\title{
Cultura de segurança do paciente e notificação de eventos adversos de equipe multiprofissional de saúde
}

\author{
Patient safety culture and adverse event notification of a multidisciplinary health team \\ Cultura de seguridad del paciente y notificación de eventos adversos de un equipo de salud
} multidisciplinario

Recebido: 17/06/2021 | Revisado: 26/06/2021 | Aceito: 28/06/2021 | Publicado: 12/07/2021

Gisele da Silveira Lemos

ORCID: https://orcid.org/0000-0001-8987-0245 Universidade Estadual do Sudoeste da Bahia, Brasil Universidade Federal de Minas Gerais, Brasil

E-mail: gisele.lemos@uesb.edu.br

Ionara Vieira Rocha da Mota

ORCID: https://orcid.org/0000-0003-2212-340X

Universidade Estadual do Sudoeste da Bahia, Brasil

E-mail: yonarocha@hotmail.com

Adriano Max Moreira Reis

ORCID: https://orcid.org/0000-0002-0017-7338

Universidade Federal de Minas Gerais, Brasil

E-mail: amreis@outlook.com

Edson Perini

ORCID: https://orcid.org/0000-0001-8833-9815

Universidade Federal de Minas Gerais, Brasil

E-mail: edsonperini@gmail.com

Mário Borges Rosa

ORCID: https://orcid.org/0000-0002-6922-8367 Instituto para Práticas Seguras no Uso de Medicamentos, Brasil

E-mail: mariobr@ismp-brasil.org

Cristiane Aparecida Menezes de Pádua

ORCID: https://orcid.org/0000-0001-7083-3188

Universidade Federal de Minas Gerais, Brasil

E-mail: cmenezespadua@gmail.com

\begin{abstract}
Resumo
Este estudo teve como objetivo compreender a cultura de segurança do paciente e notificação de eventos adversos de equipe multiprofissional de saúde de um hospital público de ensino. Estudo transversal com coleta de dados entre dezembro de 2015 a março de 2016, utilizando questionário Hospital Survey on Patient Safety Culture (HSOPSC). A amostra foi intencional constituída de profissionais de saúde envolvidos na assistência. Dos participantes (331) 44,0\% eram técnicos/auxiliares de enfermagem, $28,4 \%$ enfermeiros, $17,1 \%$ médicos e $10,5 \%$ outros. Considerando as 42 questões do instrumento um total de $5385(39,7 \%)$ respostas foram positivas, $3122(23,0 \%)$ neutras e $5066(37,3 \%)$ negativas. Observouse que $78,3 \%$ profissionais não comunicaram nenhum evento adverso (EA) nos últimos 12 meses e 52,8\% consideraram a nota de segurança do paciente como "regular". A pontuação mais positiva esteve associada com profissionais que tinham menos de um ano de trabalho no hospital $(\mathrm{p}=0,021)$, trabalham menos de 80 horas semanais $(\mathrm{p}=0,048)$, exercem cargo ou função de nível técnico $(\mathrm{p}=0,010)$, segundo grau completo $(\mathrm{p}=0,006)$ e aqueles que realizaram notificação de EA nos últimos 12 meses $(\mathrm{p}=0,016)$. A cultura de segurança do paciente no hospital de estudo ainda não está estruturada, no entanto aspectos positivos destacados podem ser utilizados como ponto de partida para a implementação de mudanças.

Palavras-chave: Segurança do paciente; Cultura organizacional; Hospitais públicos; Gestão de riscos; Equipe de assistência ao paciente.
\end{abstract}

\section{Abstract}

This study aimed to understand the patient safety culture and adverse event notification of a multiprofessional health team at a public teaching hospital. Cross-sectional study with data collection between December 2015 and March 2016, using the Hospital Survey on Patient Safety Culture questionnaire (HSOPSC). The sample was intentional, made up of all health 
professionals involved in health care. Of the participants (331), 44.0\% were nursing technicians / auxiliaries, $28.4 \%$ were nurses, $17.1 \%$ were physicians and $10.5 \%$ were others. Considering the 42 questions of the instrument, a total of 5385 $(39.7 \%)$ were positive, $3122(23.0 \%)$ were neutral and $5066(37.3 \%)$ were negative. It was observed that $78.3 \%$ of professionals reported no adverse events (AD) in the last 12 months and $52.8 \%$ considered the patient's safety score to be "regular". The most positive score was statistically related to professionals who had less than one year of work in the hospital $(\mathrm{p}=0.021)$, worked less than 80 hours a week $(\mathrm{p}=0.048)$, held a technical-level $(\mathrm{p}=0.010)$, full-time position $(\mathrm{p}=0.006)$, and those who had reported AE in the past 12 months $(\mathrm{p}=0.016)$. The safety culture of the patient in the study hospital is not yet structured, however highlighted positive aspects can be used as a starting point for implementing changes.

Keywords: Patient safety; Organizational culture; Hospitals, public; Risk management; Patient care team.

\section{Resumen}

Este estudio tuvo como objetivo comprender la cultura de seguridad del paciente y notificación de eventos adversos de un equipo multiprofesional de salud de un hospital público de enseñanza. Estudio transversal con recolección de datos entre diciembre de 2015 a marzo de 2016, utilizando cuestionario Hospital Survey on Patient Safety Culture (HSOPSC). La muestra fue intencional, constituida por todos los profesionales de la salud involucrados en la asistencia a la salud. De los participantes (331) 44,0\% eran técnicos / auxiliares de enfermería, 28,4\% enfermeros, 17,1\% médicos y 10,5\% otros. Considerando las 42 preguntas del instrumento, um total de 5385 (39,7\%), las respuestas fueron positivas, 3122 (23,0\%) neutras y 5066 (37,3\%) negativas. Se observó que el 78,3\% profesional no comunicó ningún evento adverso (EA) en los últimos 12 meses y el 52,8\% consideró la nota de seguridad del paciente como "regular". La puntuación más positiva estuvo estadísticamente relacionada con profesionales que tenían menos de un año de trabajo en el hospital ( $\mathrm{p}=0,021)$, trabajan menos de 80 horas semanales $(\mathrm{p}=0,048)$, ejercen cargo o función de nivel técnico ( $\mathrm{p}=0,0010)$, según grado completo $(\mathrm{p}=0,006)$ y aquellos que realizaron notificación de EA en los últimos 12 meses $(\mathrm{p}=0,016)$. La cultura de seguridad del paciente en el hospital de estudio aún no está estructurada, sin embargo, los aspectos positivos destacados pueden ser utilizados como punto de partida para la implementación de cambios.

Palabras clave: Seguridad del paciente; Cultura de la organización; Hospitales públicos; Gestión de riesgos; Equipo de asistencia al paciente.

\section{Introdução}

Qualidade é um requisito essencial na atenção ao paciente assistido no serviço de saúde em virtude do potencial danoso deste cuidado, com destaque para a ocorrência de incidentes, erros ou falhas na assistência, hoje considerado um grave problema de saúde mundial (Makary \& Daniel, 2016; Couto, Pedrosa \& Rosa, 2016; Silva-Batalha \& Melleiro, 2015). A segurança do paciente (SP) é uma das dimensões da qualidade em saúde e são crescentes as iniciativas para a sua promoção. Em âmbito mundial, essas iniciativas são priorizadas por formuladores de políticas, prestadores de cuidados, gestores e líderes (de Jesus Oliveira, Lima \& Provin, 2020; Andrade et al., 2018; Silva-Batalha \& Melleiro, 2015; Oliveira et al., 2014; Reis, Languardia \& Martins, 2012).

Como uma estratégia para prevenir e reduzir danos associados a assistência à saúde as instituições hospitalares buscam promover a SP como um processo cultural (Carneiro et al., 2020, Andrade et al., 2018; Tomazoni et al., 2014;). A Cultura institucional é um conjunto de ideias, percepções, comportamentos e práticas que guiam o exercício profissional dos indivíduos e equipes. Ela é um dos pilares da SP e se desenvolve com comunicação eficiente, confiança, aprendizado organizacional e compromisso da gestão com segurança (Carneiro et al., 2020; Andrade et al., 2018; Reis, Languardia \& Martins, 2012). Assim mensurar a cultura de segurança do paciente (CSP) tornou-se exigência das instituições internacionais de acreditação para conhecer a percepção da equipe em relação ao trabalho, ações administrativas e da liderança para apoiar e promover a SP, notificação de eventos adversos (EA) e outros comportamentos pessoais (de Jesus Oliveira, Lima \& Provin, 2020; El-Jardali et al., 2014).

Ultimamente a CSP vem sendo valorizada nas organizações de saúde, direcionando o comportamento dos profissionais de saúde na construção de uma visão de alta prioridade para segurança (Tomazoni et al., 2015; Silva-Batalha \& Melleiro, 2015; ElJardali et al., 2014; Reis, Languardia \& Martins, 2012). Reconhecendo sua importância, o Ministério da Saúde (MS) brasileiro 
estabeleceu, em 2013, estratégias para implementação de um Programa Nacional de Segurança do Paciente (PNSP) com propostas de mudanças culturais (Brasil, 2014).

No entanto para se instituir uma cultura que vise a SP como prioridade se faz necessário mensurar qual a cultura presente na organização de saúde. Considerando que essa discussão é recente no Brasil e uma lacuna importante no conhecimento científico diz respeito ao trabalho da equipe multiprofissional e gestores sobre o tema, este artigo tem como objetivo compreender a cultura de segurança do paciente e notificação de eventos adversos de equipe multiprofissional de saúde de um hospital público de ensino.

\section{Metodologia}

\section{Local, delineamento e amostra}

Pesquisa realizada em um hospital público de ensino, no estado da Bahia, com atendimento integral pelo Sistema Único de Saúde (SUS) e administração da Secretaria Estadual de Saúde da Bahia (Sesab). O hospital é referência em urgência e emergência e campo de ensino, pesquisa e extensão para alunos de graduação e pós-graduação. Estudo transversal abrangendo profissionais de saúde que realizam assistência direta ou indiretamente, de acordo com os seguintes critérios de inclusão: enfermeiro, médico, fisioterapeuta, farmacêutico, nutricionista, cirurgião dentista, fonoaudiólogo, psicólogo e técnico ou auxiliar de enfermagem, estar em exercício de suas funções no hospital durante o período da coleta de dados e período de admissão no hospital maior ou igual a dois meses.

Neste estudo quatrocentos e vinte quatro (424) profissionais de saúde estavam aptos a participar, com contrato ativo no momento da coleta de dados. Desses, vinte e seis profissionais estavam de licença/férias e treze não foram localizados. Dos profissionais convidados, 17 recusaram e 37 não devolveram o questionário, conforme diagrama de inclusão, Figura 1. Os profissionais que atuavam exclusivamente na clínica psiquiátrica, situada em uma área separada do hospital, não foram incluídos por dificuldades operacionais e de acesso (18 profissionais).

Figura 1 - Diagrama de inclusão dos profissionais de saúde, em um hospital público no interior da Bahia. Brasil, dezembro de 2015 a março de 2016.

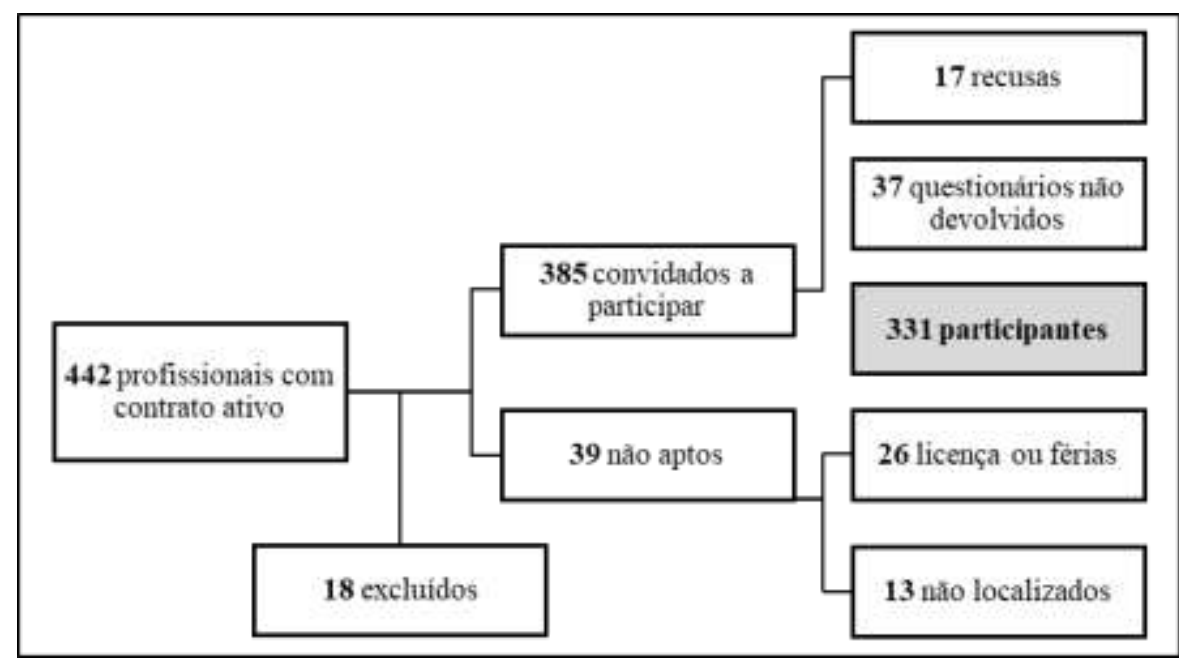

Fonte: Autores. 


\section{Instrumento de coleta e variáveis do estudo}

O questionário Hospital Survey on Patient Safety Culture (HSOPSC), criado pela Agency for Healthcare Research and Quality (AHRQ), traduzido, validado transculturalmente para o Brasil e reavaliado (Reis et al., 2019; Sorra et al., 2016; Reis et al., 2016 Reis, Languardia \& Martins, 2012;), versão em papel, foi utilizado para mensurar a CSP sob a perspectiva dos profissionais. O instrumento contém 12 dimensões (D) em escala de múltiplos itens, cada uma contendo três ou quatro questões com cinco opções de respostas numeradas (escala Likert), e 02 variáveis de resultado de item único, variáveis de resultado. Ele aborda também dez variáveis sociodemográficas e de inserção no trabalho:

- CSP no âmbito da unidade: trabalho em equipe na unidade (D1), expectativas e ações do supervisor/chefe para a promoção da SP (D2), aprendizado organizacional - melhoria contínua (D3), feedback e comunicação sobre erros (D6), abertura para a comunicação (D7), quadro de funcionários (D10), resposta não punitiva ao erro (D12),

- CSP no âmbito da organização hospitalar: apoio da gestão hospitalar para a SP (D4), trabalho em equipe entre as unidades hospitalares (D9), transferências internas e passagem de plantão (D11);

- dimensões de resultado: frequência de eventos comunicados (D8) e percepção geral de SP (D5).

- variáveis de resultado: A primeira variável 'número de eventos comunicados nos últimos 12 meses' comunicados refere-se aos incidentes que resultaram em danos à saúde informados pelo profissional ao seu supervisor ou chefia, tendo como opções de nenhum a mais de 21 eventos comunicados. A segunda é referente à percepção do profissional sobre a SP por meio da atribuição de uma nota (excelente, muito boa, regular, ruim e muito ruim) para SP na sua área ou unidade de trabalho (Sorra et al., 2016; Reis et al., 2016; Reis, Languardia \& Martins, 2012).

Foram consideradas variáveis dependentes: respostas positivas de cada uma das dimensões, nota de segurança do paciente (NSP) e notificação de EA nos últimos 12 meses; e independentes: variáveis sociodemográficas, de trabalho, cargo ou função, área ou setores hospitalares e notificação de eventos nos últimos 12 meses.

\section{Coleta de dados}

A coleta de dados ocorreu entre os meses de dezembro de 2015 a março de 2016. O projeto foi apresentado aos profissionais durante os horários de trabalho no hospital, conforme as escalas fornecidas pela coordenação. Os presentes eram convidados a participar e assinar o termo de consentimento livre esclarecido. O questionário era entregue e recolhido ao final do plantão ou em plantões subsequentes. Profissionais procurados por cinco vezes ou mais para a entrega do questionário respondido, sem sucesso, foram classificados como "não devolvidos"; profissionais não presentes em dias de trabalho da escala por cinco vezes ou mais foram designados "não localizados".

\section{Análise dos dados}

Os questionários foram revisados quanto ao seu preenchimento e em caso de preenchimento de uma única opção para uma seção este foi excluído (por haver questões invertidas, perspectivas positivas e negativas). Os dados foram agrupados e tabulados no software EpiData, versão 3.1 2008, e analisados no software SPSS, versão 21.0, 2012 (IBM Corp, Armonk, Estados Unidos). A análise descritiva foi apresentada por meio de frequências (absoluta e relativa), médias e desvio-padrão. O escore de cada dimensão foi descrito por meio da distribuição percentual das respostas positivas, negativas e neutras dos itens de cada dimensão. As dimensões foram classificadas em duas áreas: áreas de força, 75\% (desejável) ou mais de respostas positivas; e áreas críticas, 50\% ou menos de respostas positivas (Sorra et al., 2016). 
A avaliação das respostas foi realizada conforme as orientações da AHRQ (Sorra et al., 2016). Elas foram, agrupadas em três categorias:

- respostas positivas: concordo totalmente / concordo ou frequentemente / sempre para as perguntas com perspectiva positiva e discordo totalmente / discordo ou nunca / raramente para as perguntas com perspectiva negativa;

- respostas negativas: discordo totalmente/discordo ou nunca/raramente para perguntas com perspectiva positiva, ou usando concordo totalmente/concordo, sempre/ frequentemente para perguntas com perspectiva negativa;

- respostas neutras: não concordo/ nem discordo e as vezes.

Associações estatísticas entre respostas positivas, NSP e eventos notificados nos últimos 12 meses com variáveis sociodemográficas e de trabalho foram analisadas pelo Qui-quadrado de Pearson com correção de Bonferroni. Foi considerado o nível de significância de $\mathrm{p}<0,05$.

\section{Questões éticas}

O estudo foi realizado conforme a Resolução 466/2012 do Conselho Nacional de Saúde, MS. O projeto foi aprovado pelo Comitê de Ética em Pesquisa da Universidade Federal de Minas Gerais, CAAE: 49477015.6.0000.5149.

\section{Resultados}

Dos profissionais aptos a participarem, 86,0\% (331/385) responderam ao questionário (37 não devolveram e 17 recusaram), taxa de resposta $89,9 \%$. A não participação, segundo as categorias profissionais, foi assim distribuída: 11,6\% dos técnicos de enfermagem, $14,9 \%$ dos enfermeiros e $24,3 \%$ dos médicos. Entre os participantes $74,1 \%$ declararam-se do sexo feminino e idade média de 38,7 (11,8) anos. Considerando o cargo ou função no hospital, 44,0\% eram técnicos ou auxiliares de enfermagem, 28,5\% enfermeiros, $17,1 \%$ médicos, 5,8\% fisioterapeutas ou fonoaudiólogos, 1,8\% nutricionistas e 2,8\% farmacêuticos, psicólogos ou cirurgiões dentista. Cerca da metade $(51,4 \%$ ) possuía pós-graduação (lato sensu ou strictu sensu). O tempo médio de formado era de 14,2 $(12,1)$ anos, e 30,1\% trabalhava entre 6 a 10 anos na especialidade ou profissão. Aproximadamente metade dos profissionais $(45,4 \%)$ relataram ter entre 1 a 5 anos de serviço no hospital e 52,1\% na área ou unidade que atua; 66,6\% dedicavam 20 a 30 horas de trabalho por semana no hospital e 94,8\% mantinham interação ou contato direto com pacientes. As unidades de inserção profissional no hospital mais citadas foram emergência e clínica cirúrgica, 25,0 e 17,7\%, respectivamente.

Para os 42 itens de respostas, agrupadas nas 12 D, os percentuais médios das respostas podem ser vistos na Figura 1. Nenhuma dimensão pode ser classificada como área de força, porém algumas se destacaram com índices de avaliação positiva mais altos. Dentre as dimensões, o maior valor total de respostas positivas, considerando as quatro questões, $(793 ; 61,2 \%)$ foi observado na D1, "Trabalho em equipe na unidade", que trata do apoio e do respeito entre os funcionários e do trabalho realizado em equipe na área ou unidade. O item mais bem avaliado foi "Nesta unidade, as pessoas se tratam com respeito" - com 77,7\% (251) dos profissionais concordando com a afirmação, indicando um ponto positivo na CSP institucional.

A dimensão que caracteriza atitudes do supervisor ou chefia relacionada à promoção da segurança (“Expectativas e ações do supervisor/chefia para a promoção da SP") foi a segunda em respostas positivas (773; 59,3\%). Reforça essa posição o fato de que 222 (67,9\%) profissionais discordaram da afirmativa "O meu supervisor/chefe não dá atenção suficiente aos problemas de SP que acontecem repetidamente", item de maior destaque entre os três que a compõe. Todas as demais dimensões receberam percentual menor que $50 \%$ para as respostas positivas. 
A D4, "Apoio da gestão hospitalar para segurança do paciente", e D5, "Percepção geral da SP", receberam os menores percentuais de respostas positivas, 28,2 (277) e 29,2\% (667), respectivamente, sendo classificadas como áreas críticas. Dos quatro itens que a compõe a D5, o pior avaliado foi justamente a percepção da existência de problemas de segurança no setor ("Nesta unidade temos problemas de SP"), com 65,3\% (213) dos profissionais concordando com a afirmação.

Figura 1 - Distribuição percentual das respostas positivas, neutras e negativas das doze dimensões da cultura de segurança do paciente (42 questões), em um hospital público no interior da Bahia. Brasil, dezembro de 2015 a março de 2016 (N=13573).

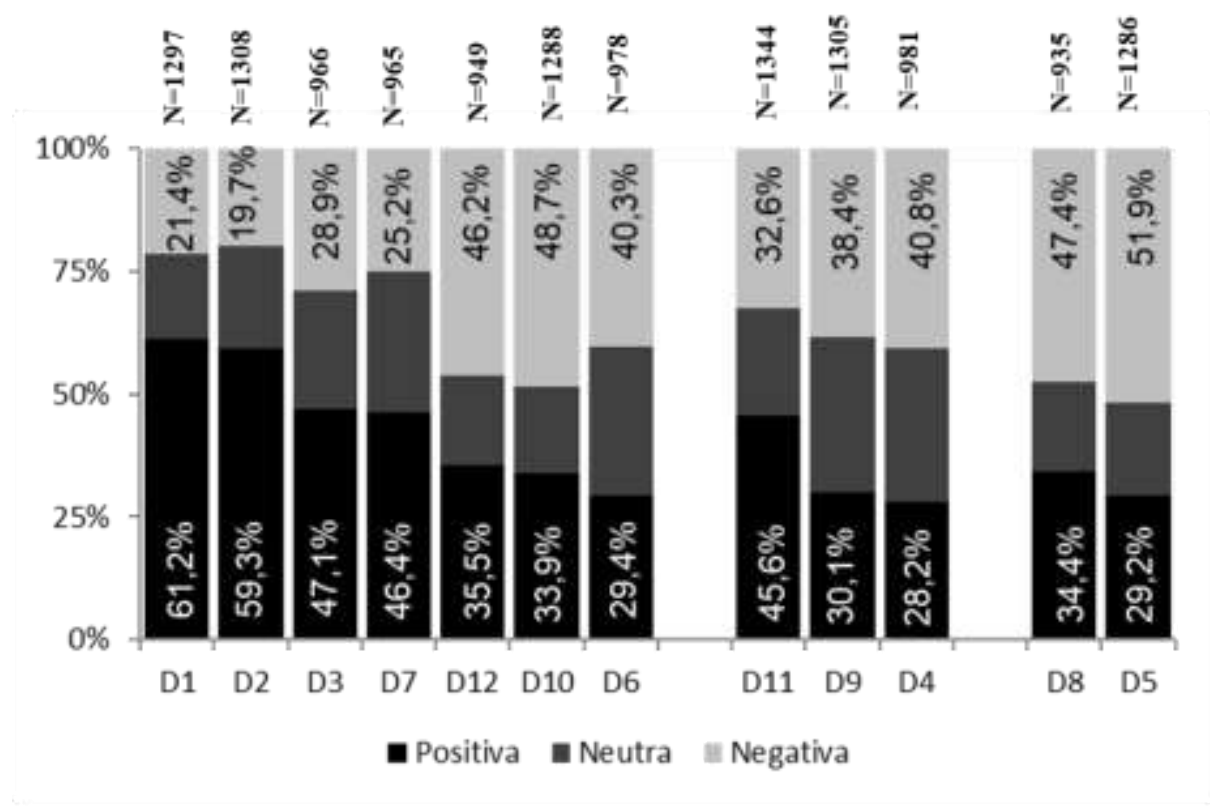

Dimensōes:

- Cultura de Segurança no âmbito da unidade hospitalar: D1: trabalho em equipe na unidade; D2: expectativas e açōes do supervisor/chefe para promoção da segurança do paciente; D3: aprendizado organizacional - melhoria continua; D7: abertura para comunicação; D12: resposta năo punitiva ao erro; D6: feedback e comunicaçào sobre erros.

- Cultura de Segurança no âmbito da organizaçào: D11: transferências internas e passagem de plantào; D10: quadro de funcionários; D9: trabalho em equipe entre as unidades hospitalares; D4: apoio da gestão hospitalar para segurança do paciente;

- Variáveis de resultado: D8: frequência de eventos comunicados; D5: percepção geral da segurança

do paciente

A maioria dos participantes não comunicou nenhum EA nos últimos 12 meses, Figura 2.A, soma-se a esse resultado o fato de que nos itens da D8 (Frequência de eventos comunicados) as respostas negativas prevaleceram em relação as positivas para a notificação de eventos ou quase eventos, com 47,9\% e 34,4\%, respectivamente. A percepção sobre a SP foi atribuída com a nota "Regular" pela maioria dos profissionais (161; 52,8\%) (Figura 2.B). 
Figura 2 - (A) Distribuição percentual do número de eventos comunicados nos últimos 12 meses (B) Distribuição percentual sobre a percepção da segurança do paciente, em um hospital público no interior da Bahia. Brasil, dezembro de 2015 a março de 2016.
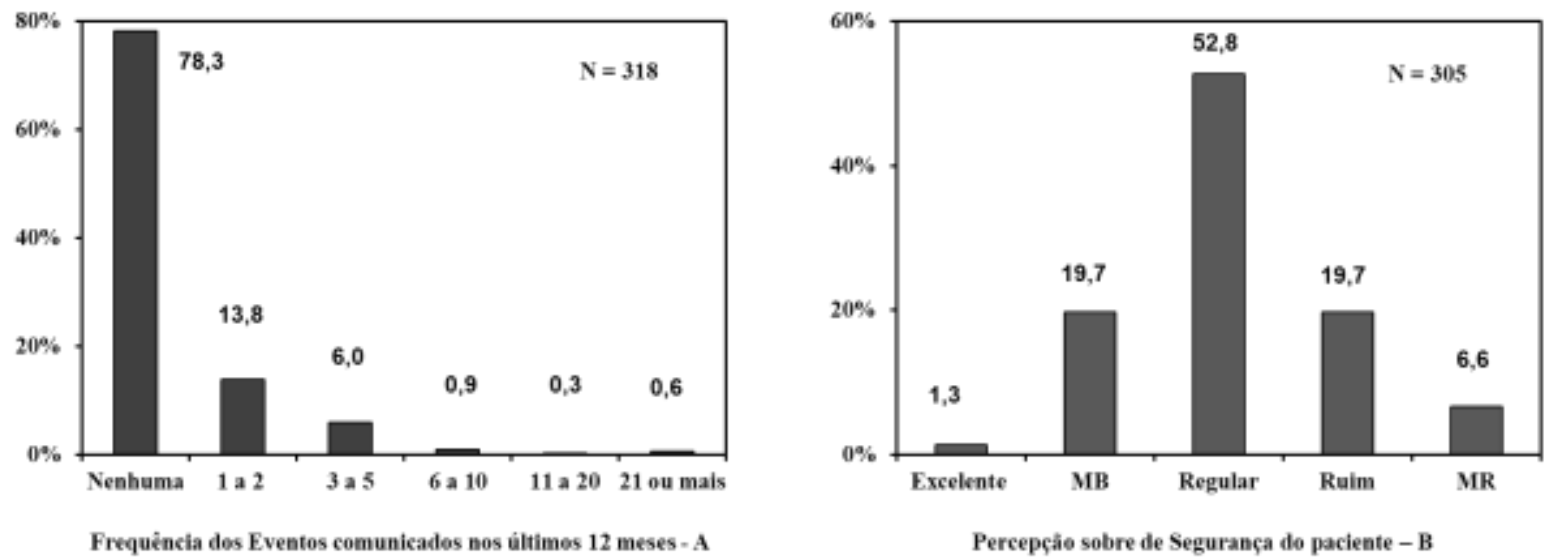

MB: muito bom, MR: muito ruim. Fonte: Autores.

Os profissionais com menos de um ano de trabalho no hospital responderam mais positivamente, enquanto aqueles com carga horária superior a 80 horas semanais foram os que menos pontuaram positivamente. Em relação ao cargo/ função no hospital e formação acadêmica, técnicos/ auxiliares de enfermagem e segundo grau completo declararam mais respostas positivas. Referindose a notificação de eventos nos últimos 12 meses o número de respostas positivas foi maior para aqueles profissionais que realizaram alguma notificação nesse período (Tabela 1).

Técnicos/ auxiliares de enfermagem responderam com percepção mais positiva (excelente e muito bom) para a NSP enquanto os médicos responderam com percepção menos positiva, resultado ratificado para o grau de instrução uma vez que profissionais com segundo grau completo responderam mais positivamente a NSP do que os de nível superior/ pós graduação (Tabela 2). 
Tabela 1 - Respostas Positiva para as 42 questões, das 12 dimensões sobre fatores da cultura de segurança, de acordo com características de trabalho, sociodemográficas e notificação de eventos nos últimos 12 meses em um hospital público. Bahia, Brasil, dezembro de 2015 a março de 2016.

\begin{tabular}{|c|c|c|}
\hline $\begin{array}{l}\text { Características de trabalho, sociodemográficas e } \\
\text { dimensão de resultado }\end{array}$ & Respostas Positivas \% & *p-valor \\
\hline Tempo de trabalho no hospital (anos) & $\mathrm{N}=5362$ & 0,021 \\
\hline$<1$ & 46,8 & \\
\hline $1-5$ & 40,5 & \\
\hline $6-10$ & 37,8 & \\
\hline $11-15$ & 25,6 & \\
\hline $16-20$ & 22,8 & \\
\hline+21 anos & 41,4 & \\
\hline Tempo de trabalho na unidade (anos) & $\mathrm{N}=5261$ & 0,201 \\
\hline$<1$ & 42,2 & \\
\hline $1-5$ & 38,7 & \\
\hline $6-10$ & 40,2 & \\
\hline $11-15$ & 35,2 & \\
\hline $16-20$ & 26,6 & \\
\hline+21 & 46,8 & \\
\hline Horas por semana no hospital & $\mathrm{N}=5284$ & 0,048 \\
\hline$<20$ horas & 37,2 & \\
\hline 20 a 39 horas & 39,4 & \\
\hline 40 a 59 horas & 41,5 & \\
\hline 60 a 79 horas & 36,0 & \\
\hline 80 a 99 horas & 32,7 & \\
\hline Cargo /função neste hospital & $\mathrm{N}=\mathbf{5 3 1 0}$ & 0,010 \\
\hline Médico & 27,0 & \\
\hline Enfermeiro & 38,1 & \\
\hline Fisioterapeuta & 33,6 & \\
\hline Técnico/Auxiliar de Enfermagem & 46,6 & \\
\hline Outros** & 39,0 & \\
\hline Grau de instrução & $\mathrm{N}=\mathbf{5 3 2 3}$ & 0,006 \\
\hline $2^{\circ}$ grau completo & 48,6 & \\
\hline Superior completo & 42,1 & \\
\hline Pós-graduação & 34,8 & \\
\hline Notificação de eventos adversos & $\mathrm{N}=5139$ & 0,016 \\
\hline Sim & 40,8 & \\
\hline Não & 39,5 & \\
\hline
\end{tabular}

* Teste de qui-quadrado de Pearson. **Outros: farmacêutico, psicólogo, cirurgião dentista e nutricionistas. Fonte: Autores. 
Tabela 2 - Notas de Segurança, dada à instituição hospitalar pelos colaboradores, e Notificação de eventos adversos por cargo, grau de instrução e características de trabalho em um hospital público. Bahia, Brasil, dezembro 2015 a março 2016.

\begin{tabular}{|c|c|c|c|c|c|c|c|}
\hline \multirow{2}{*}{$\begin{array}{l}\text { Características de trabalho e } \\
\text { sociodemográficas }\end{array}$} & \multicolumn{3}{|c|}{ Nota de seguranca } & \multicolumn{4}{|c|}{ Notificação } \\
\hline & Muito bom/ Bom & Regular & $\begin{array}{l}\text { Muito ruim/ } \\
\text { Ruim }\end{array}$ & p-valor & Não notificou & Notificou & p-valor \\
\hline \multicolumn{8}{|l|}{ Tempo de trabalho no hospital } \\
\hline$<1$ ano & 8 & 13 & 5 & 0,580 & 24 & 3 & 0,468 \\
\hline $1-5$ anos & 32 & 68 & 35 & & 110 & 33 & \\
\hline $6-10$ anos & 14 & 51 & 25 & & 70 & 23 & \\
\hline 11-15 anos & 2 & 3 & 2 & & 7 & 0 & \\
\hline $16-20$ Anos & 1 & 2 & 4 & & 5 & 2 & \\
\hline+21 anos & 6 & 22 & 9 & & 31 & 8 & \\
\hline \multicolumn{8}{|l|}{ Tempo de trabalho na unidade } \\
\hline$<1$ ano & 13 & 26 & 12 & 0,901 & 41 & 10 & 0,976 \\
\hline $1-5$ anos & 30 & 83 & 42 & & 130 & 36 & \\
\hline $6-10$ anos & 13 & 36 & 14 & & 47 & 15 & \\
\hline 11-15 anos & 2 & 3 & 3 & & 6 & 2 & \\
\hline 16 - 20 Anos & 1 & 3 & 4 & & 7 & 1 & \\
\hline+21 anos & 3 & 8 & 5 & & 15 & 4 & \\
\hline \multicolumn{8}{|l|}{ Horas por semana no hospital } \\
\hline$<20$ horas por semana & 5 & 13 & 7 & 0,268 & 21 & 6 & 0,718 \\
\hline 20 a 39 horas por semana & 34 & 112 & 58 & & 171 & 42 & \\
\hline 40 a 59 horas por semana & 21 & 30 & 12 & & 49 & 19 & \\
\hline 60 a 79 horas por semana & 0 & 2 & 1 & & 3 & 1 & \\
\hline 80 a 99 horas por semana & 1 & 2 & 2 & & 3 & 1 & \\
\hline \multicolumn{8}{|l|}{ Cargo /função neste hospital } \\
\hline Médico & $6^{\mathrm{a}}$ & $23^{\mathrm{a}}$ & $25^{\mathrm{b}}$ & $<0,001$ & 46 & 9 & 0,641 \\
\hline Enfermeiro & $12^{\mathrm{a}}$ & $49^{\mathrm{a}}$ & $26^{\mathrm{a}}$ & & 73 & 22 & \\
\hline Fisioterapeuta & $4^{\mathrm{a}}$ & $10^{\mathrm{a}}$ & $4^{\mathrm{a}}$ & & 12 & 6 & \\
\hline Técnico/Auxiliar de Enfermagem & $39^{\mathrm{a}}$ & $75^{\mathrm{a}}$ & $19^{\mathrm{b}}$ & & 109 & 29 & \\
\hline Outros & $2^{\mathrm{a}}$ & $3^{\mathrm{a}}$ & $6^{\mathrm{a}}$ & & 7 & 2 & \\
\hline \multicolumn{8}{|l|}{ Grau de instrução } \\
\hline $2^{\circ}$ grau completo & $17^{\mathrm{a}}$ & $39^{\mathrm{a}}$ & $5^{b}$ & 0,002 & 44 & 18 & 0,114 \\
\hline Superior completo & $19^{\mathrm{a}}$ & $44^{\mathrm{a}}$ & $19^{\mathrm{a}}$ & & 74 & 13 & \\
\hline Pós-graduação & $27^{\mathrm{a}}$ & $77^{\mathrm{a}}$ & $56^{\mathrm{b}}$ & & 129 & 37 & \\
\hline
\end{tabular}

* Teste de qui-quadrado de Pearson. Outros: Farmacêutico, Nutricionista, Cirurgião dentista e Psicólogo. a,b: correção de bonferroni. Source: Authors. 


\section{Discussão}

O presente estudo contribui para a compreensão da CSP no contexto hospitalar, com avaliação de uma equipe multiprofissional de saúde. Percebe-se que o cargo, grau de instrução e tempo e horas de trabalho no hospital influenciam na percepção dos profissionais sobre SP.

Não identificamos, na percepção dos profissionais, uma área de força para a SP, o que nos leva a entender que a instituição não apresenta uma CSP bem estruturada e implementada, esse padrão de resultado com ausência de dimensões fortalecidas e itens que se destacam positivamente foi encontrado em estudos (de Jesus Oliveira, Lima \& Provin, 2020; Silva et al., 2019; Cruz et al., 2018). No entanto, o número de respostas positivas para algumas dimensões, como "Trabalho em equipe na unidade" e "Expectativas e ações do supervisor/chefia para a promoção da SP", pode significar avanços na formação dessa cultura, que podem estar em processo de crescimento e virem a se tornar áreas fortes.

As dimensões citadas anteriormente também foram destaque em hospitais na Espanha, Arábia Saudita, China, Tunísia, EUA e Brasil. Em hospitais internacionais observa-se um maior número de dimensões pontuadas positivamente, comparado com o Brasil, devido a singularidade cultural que deve ser levada em consideração neste tipo de avaliação e no Brasil o incentivo a CSP ser recente (considerando data de publicação do PNSP) (de Jesus Oliveira, Lima \& Provin, 2020; Cheikh et al., 2016; ElJardali et al., 2014; Gama, Oliveira \& Hernandez, 2013; Nie et al., 2013; Sorra et al., 2012).

Em uma revisão sobre CS na aviação e indústrias de alto risco a dimensão "Trabalho em equipe na unidade" foi considerada a chave para exercer uma força positiva na percepção de segurança, pois pode indicar uma boa relação dentro da unidade com seus supervisores diretos, podendo ser utilizada como apoio nas mudanças e intervenções de melhora da segurança (Wang \& Tao, 2017). A promoção de uma CSP implica estabelecer intervenções enraizadas nos princípios da liderança, do trabalho em equipe e na mudança de comportamento (Macedo et al., 2016).

A pontuação positiva da dimensão "Expectativas e ações do supervisor/chefia para a promoção de SP" demonstra a percepção dos trabalhadores quanto ao apoio da gestão direta para a SP oferecendo um clima de trabalho propício em que todos se ajudam. Em outros estudos essa dimensão também aparece como destaque positivo e os escores obtidos se aproximam do encontrado no presente estudo (Andrade et al., 2018; Wang \& Tao, 2017; Tostes, Haracemiw \& Ma, 2016; Silva-Batalha \& Melleiro, 2015; Oliveira et al., 2014; Gama, Oliveira \& Hernandez, 2013; Nie et al., 2013; Sorra et al., 2012).

A cultura organizacional é positivamente correlacionada com o comportamento do líder. Este tem influência em comportamentos, valores e crenças de seus colaboradores, contribuindo para o fortalecimento de uma cultura organizacional segura (de Jesus Oliveira, Lima \& Provin, 2020; Wang \& Tao, 2017). A ideia é que o líder desempenhe um papel do modelo a ser seguido, que por meio de suas preocupações, molde a cultura de uma unidade ou organização, demonstrando suas preferências e comunicando o que considera importante. Essas preferências tornar-se-ão as preocupações dos colaboradores, visto que poderão representar a concessão de recursos, recompensas ou punições (Minuzzi et al., 2016).

Para as áreas críticas a pior distribuição geral nas dimensões foi "Percepção geral de SP" que pode demonstrar insuficiência em relação as atividades da gerência geral do hospital dirigidas a melhorar a segurança, assim como demonstram dificuldades em reconhecer a priorização por parte da gestão quanto a aspectos que envolvem SP (Macedo et al., 2016; Gama, Oliveira \& Hernandez, 2013). Em outros trabalhos essa dimensão também aparece entre as áreas críticas relatadas por profissionais de saúde (Cheikh et al., 2016; Silva-Batalha \& Melleiro, 2015; Gama, Oliveira \& Hernandez, 2013).

A literatura mostra que existe uma percepção diferenciada da CSP entre organizações hospitalares podendo variar entre profissionais de saúde dependendo do cargo, unidade de trabalho e tempo de experiência (Silva et al., 2019; Cheikh et al., 2016; Tomazoni et al., 2014). Neste estudo verificou-se que profissionais com mais tempo de trabalho na instituição optaram por menos respostas positivas, como em outro estudo brasileiro, isso pode ser justificado pelo fato de os indivíduos desenvolverem hábitos bons ou ruins de acordo com o clima de segurança da instituição em que estão inseridos (Tomazoni et al., 2014). Houve também 
diferença no número de respostas positivas dadas pelos profissionais de segundo grau completo, quando comparado com os de curso superior e pós-graduados, demonstrando que a visão diferenciada sobre segurança pode ser resultado de uma qualificação diferenciada e a ocupação de cargos com responsabilidades diferentes. Estudo na Arábia Saudita demonstrou associação entre maiores graus de instrução com menores níveis de SP (El-Jardali et al., 2014).

Estudos demonstraram a relação entre melhora na medida dos escores de CSP e implantação de programas de qualidade, treinamento e capacitação permanente, com redução das taxas de EA associados ao cuidado (Golle et al., 2018; Xie et al., 2017). Nos desafios para o desenvolvimento de uma cultura é fundamental ouvir as opiniões e preocupações dos profissionais de saúde no que se tange a SP, objetivando obter um diagnóstico das forças e fragilidades da Organização de saúde.

Não foi percebida na Organização hospitalar estudada uma cultura de notificação de EA, onde maioria dos profissionais não realizou nenhuma notificação nos últimos 12 meses. Outros trabalhos realizados em hospitais brasileiros e internacionais também demonstram uma baixa notificação de EA pelas equipes, mostrando a vulnerabilidade do processo de notificação (Arboit et al., 2020; de Jesus Oliveira, Lima \& Provin, 2020; Andrade et al., 2016; Silva-Batalha \& Melleiro, 2015; Tomazoni et al., 2015; Sorra et al., 2012). Faz-se, portanto, necessário encorajar os profissionais de saúde, uma vez que conhecendo a importância do processo de notificação para o aprendizado organizacional, essa torna-se culturalmente apropriada.

Não se verificou associação estatística entre a notificação de EA e tempo de trabalho, cargo/ função e grau de instrução, diferente de estudo nacional e internacional (El-Jardali et al., 2014; Tomazoni et al., 2015). Isso pode ser justificado pelo fato de inexistir na instituição estudada uma estratégia para o relato de EA, até o período da coleta de dados. Além disso a D12, resposta não punitiva ao erro, teve um baixo escore positivo o que pode ser resultado de uma cultura de punição, e para que a cultura justa se estabeleça como CS está o conhecimento de todos os profissionais do que é aceitável e o que a organização não pode tolerar no que se refere aos erros com os pacientes.

Em uma CSP positiva a notificação é uma ferramenta reativa utilizada para a gestão do risco. A subnotificação dos "eventos" impede uma análise para determinar as causas de origem das falhas no sistema (Arboit et al., 2020; de Jesus Oliveira, Lima \& Provin, 2020; Brasil, 2014). Além disso, os dados notificados geram a aprendizagem contínua, indução do enfrentamento dos problemas identificados e adoção de medidas gerenciadas em base ao risco com o intuito de evitar que danos aos pacientes venham a se repetir (Brasil, 2014).

Apesar das áreas de forças evidenciadas, a maioria dos profissionais elencou a NSP como "regular". Com relação às respostas essas condições podem ser de fato regulares ou tais respostas podem revelar o desconhecimento dos entrevistados sobre o que é necessário para que o hospital em questão seja um ambiente de assistência seguro. As escolhas pelas NSP também foram diferentes de acordo com o cargo/ função, sendo os técnicos/ auxiliares de enfermagem e os médicos os que tiveram uma percepção mais positiva (excelente/ muito boa) e menos positiva (ruim/ muito ruim), respectivamente. Estudo espanhol demonstrou que enfermeiros responderam mais positivamente em relação a nota dos médicos (Gama, Oliveira \& Hernandez, 2013) e em estudo Chinês maioria dos profissionais declararam uma NSP positiva indicando que este país está à frente no desenvolvimento da sua CSP (Nie et al., 2013).

Sobre as limitações deste estudo, podemos destacar a limitação espacial de pesquisa realizada em apenas uma instituição hospitalar. Apesar de os dados não poderem ser extrapolados diretamente para outros estabelecimentos brasileiros, a literatura nacional nos mostra que a realidade aqui apresentada é bastante comum no país. Destaca-se também como limitação a não avaliação da CSP pelas unidades hospitalares, não sendo possível a identificação de subculturas. No entanto, salientamos o uso de um questionário internacional bem aceito, traduzido e validado para o Brasil.

Os resultados desta pesquisa demonstram muitas áreas para melhoria, particularmente quando comparado com outros países. No entanto o estímulo a SP em nosso país tem progredido, com incentivo ao treinamento e educação permanente, sendo que os dados servem de base para a tomada de decisão de gestores e núcleo de segurança do paciente. 


\section{Conclusão}

Ao mensurar a cultura de segurança do paciente da equipe multiprofissional de saúde dessa Organização hospitalar foi possível identificar fatores que favorecem e barreiras que interferem na CSP da instituição, possibilitando um planejamento com foco nos melhores resultados. Assim contribuindo para a consolidação de um debate sobre o tema em instituições de saúde.

\section{Referências}

Andrade, L. E. L, Lopes, J. M., Souza Filho, M. C. M., Vieira Júnior, R. F., Farias, L. P. C., Santos, C. C. M. \& Gama, Z. A. S. (2018). Cultura de segurança do paciente em três hospitais brasileiros com diferentes tipos de gestão. Ciência \& Saúde Coletiva, 23(1), 161-172.

Arboit, E. L., Bellini, G., Schütz, C. R., Moraes, M. C. S., Kinalski, S. da S. \& Barcellos, R. de A. (2020). Patient safety culture in the multiprofessional perspective. Research, Society and Development, 9(5), e125953088. 10.33448/rsd-v9i5.3088.

Ministério da Saúde (BR). (2014). Documento de referência para o Programa Nacional de Segurança do Paciente. Brasília.

Carneiro, A. S., Andolhe, R., Lanes, T. C. \& Magnago, T. S. B. de S. Patient safety culture in hospital environment: trends in brazilian production. Research, Society and Development, 9(7), e963975132, 2020. 10.33448/rsd-v9i7.5132.

Cheikh, A. B., Bouafia, N., Mahjoub, M., Ezzi, O., Nouira, A. \& Njah, M. (2016). Patient's safety culture among Tunisian healthcare workers: results of a cross sectional study in university hospital. Pan African Medical Journal, 24, 299.

Couto, R. C., Pedrosa, T. M. G., Rosa, M. B. (2016). Erros acontecem. A força da transparência para o enfrentamento dos eventos adversos assistenciais em pacientes hospitalizados. Belo Horizonte. Instituto de Estudos de Saúde Suplementar. Universidade Federal de Minas Gerais.

Cruz, E. D. A., Rocha, D. J. M., Mauricio, A. B., Ulbrich, F. S., Batista, J. \& Maziero, E. C. S (2018). Cultura de segurança entre profissionais de saúde em hospital de ensino. Cogitare Enfermagem, 23(1), e50717. http://dx.doi.org/10.5380/ce.v23i1.50717

de Jesus Oliveira, F. M., Lima, D. M. \& Provin, M. P. (2020). The culture of patient safety in an emergency and emergency public hospital. Research, Society and Development, 9(4), 6. 10.33448/rsd-v9i4.2650.

El-Jardali, F., Sheikh, F., Garcia, N. A., Jamal, D. \& Abdo, A. (2014). Patient safety culture in a large teaching hospital in Riyadh: baseline assessment, comparative analysis and opportunities for improvement. BMC Health Services Research, 14,122.

Gama, Z. A. S., Oliveira, A. C. S. \& Hernandez, P. J. S. (2013). Cultura de seguridad del paciente y factores asociados en una red de hospitales públicos Españoles. Cad. Saúde Pública. 29(2), 283-293.

Golle, L., Ciotti, D., Herr, G. E. G., Aozane, F., Schmidt, C.R. \& Kolankiewicz, A. C. B. (2018). Cultura de segurança do paciente em hospital privado. Rev. Fund Care Online, 10(1), 85-89. 10.9789/2175-5361.2018.v10i1.85-89

Macedo, T. R., Rocha, P. K., Tomazoni, A., Souza, S., Anders, J. C. \& Davis, K. (2016). The culture of patient safety from the perspective of the pediatric emergency nursing team. Rev Esc Enferm USP, 50(5), 757-763.

Makary, M. A. \& Daniel, M. (2016). Medical error - the third leading cause of death in the US. BMJ, 353(2139), 1-5.

Minuzzi, A. P., Salum, N. C., Locks, M. O. H., Amante, L. N. \& Matos, E. (2016). Contributions of healthcare staff to promote patient safety in intensive care. Esc Anna Nery, 20(1), 121-129.

Nie, Y., Mao, X., Cui, H., He, S., Li, J. \& Zhang, M. (2013). Hospital survey on patient safety culture in China. BMC Health Services Research, $13,228$.

Oliveira, R. M., Leitão, I. M. T. A., Silva, L. M. S., Figueiredo, S. V., Sampaio, R. L. \& Gondim, M. M. (2014). Strategies for promoting patient safety: from the identification of the risks to the evidence-based practices. Esc Anna Nery, 18(1),122-129.

Reis, C. T., Laguardia, J. \& Martins, M. (2012). Adaptação transcultural da versão brasileira do Hospital Survey on Patient Safety Culture: etapa inicial. Cad. Saúde Pública, 28(11), 2199-2210.

Reis, C. T., Laguardia, J., Barros, C. G. B., Andreoli, P. B. A \& Martins, M. (2019). Reliability and validity of the Brazilian version of the HSOPSC: a reassessment study. Cad. Saúde Pública, 35(8), e00246018

Reis, C. T., Laguardia, J., Vasconcelos, A. G. G. \& Martins, M. (2016). Reliability and validity of the Brazilian version of the Hospital Survey on Patient Safety Culture (HSOPSC): a pilot study. Cad. Saúde Pública, 32(11), e00115614.

Silva, A. E. B. C., Cavalcante, R. G. F., Lima, J. C., Sousa, M. R. G., Sousa, T. P. \& Nunes, R. L. S. (2019). Evaluation of the patient safety climate in hospitalization units: a cross-sectional study. Rev Esc Enferm USP, 53, e03500. http://dx.doi.org/10.1590/S1980-220X2018027203500

Silva-Batalha. E. M. S. \& Melleiro, M. M. (2015). Patient safety culture in a teaching hospital: differences in perception existing in the different scenarios of this institution. Texto Contexto Enferm., 24(2), 432-41.

Sorra, J. S., Famolaro, T., Dyer, N., Nelson, D. \& Smith, S. A. (2012). Hospital survey on patient safety culture 2012 user comparative database report. (Prepared by Westat, Rockville, MD, under Contract No. HHSA 290200710024C). Rockville, MD: Agency for Healthcare Research and Quality. 
Research, Society and Development, v. 10, n. 8, e27410817291, 2021

(CC BY 4.0) | ISSN 2525-3409 | DOI: http://dx.doi.org/10.33448/rsd-v10i8.17291

Sorra, J., Gray, L., Streagle, S., Famolaro, T., Yount, N. \& Behm, J. (2016). AHRQ Hospital Survey on Patient Safety Culture: User's Guide. (Prepared by Westat, under Contract No. HHSA290201300003C). AHRQ Publication No. 15-0049-EF (Replaces 04-0041). Rockville, MD: Agency for Healthcare Research and Quality. January 2016. https://www.ahrq.gov/sites/default/files/wysiwyg/professionals/quality-patient-safety/patientsafetyculture/hospital/userguid e/hospcult.pdf

Tomazoni, A., Rocha, P. K., Kusahara, D. M., Souza, A. I. J. \& Macedo, T. R. (2015). Evaluation of the patient safety culture in neonatal intensive care. Texto Contexto Enferm., 24(1), 161-9.

Tomazoni, A., Rocha, P. K., Souza, S., Anders, J. C. \& Malfussi, H. F. C. (2018). Patient safety culture at neonatal intensive care units: perspectives of the nursing and medical team. Rev. Latino-Am. Enfermagem, 22(5),755-63.

Tostes, M. F. P., Haracemiw, A. \& Ma, L. D. (2016). Surgical Safety Checklist: considerations on institutional policies. Esc Anna Nery. 20 (1), $203-209$.

Wang, M. \& Tao, H. (2017). How does patient safety culture in the surgical departments compare to the rest of the county hospitals in xiaogan city of China? Int J Environ Res Public Health, 14(10), 1123. 10.3390/ijerph14101123

Xie, J. F, Ding, S. Q., Zhong, Z. Q., Zeng, S. N., Qin, C. X., Yi, Q. F., Li-Na. G \& Jian, Z. (2017). A safety culture training program enhanced the perceptions of patient safety culture of nurse managers. Nurse Educ Pract., 27, 128-133. 